\title{
Readying nurses for clinical practice: Protecting students during an outbreak of Middle Eastern-Coronavirus in Saudi Arabia
}

\author{
Bridget V. Stirling , Jennie Harmston \\ Nursing Department, Princess Nourah University, Riyadh, Saudi Arabia.
}

Received: July 21, 2014

DOI: $10.5430 /$ jnep.v5n2p40
Accepted: October 18, $2014 \quad$ Online Published: November 16, 2014

URL: http://dx.doi.org/10.5430/jnep.v5n2p40

\begin{abstract}
Background: Saudi Arabia is experiencing an outbreak of Middle Eastern-Coronavirus (MERS-CoV) both in the hospitals and in the community. The MERS-CoV kills about $40 \%$ of those infected and has been detected in 22 different countries worldwide. The Faculty of Nursing at the world's largest female-only university banded together to create an education program to prevent exposure and ultimately infection of students and staff from MERS-CoV.

Methods: This study included two questionnaires given before and after a series of educational workshops to understand what students knew, what they learned, what was happening at the clinical setting, and where they were getting their information. The purpose was to answer questions and to inform the nursing college community about best practices for disease transmission prevention. A pre and post-questionnaire was given after approval from the college ethical committee. Summary statistics are presented as percentages of the participant sample that responded to each question. $T$-tests were performed to assess the difference between means of the pre and post-test.
\end{abstract}

Results: This study showed that baseline knowledge was low before the educational workshop sessions. While knowledge increased, anxiety decreased ( $p$ less than 0.05 ), and intention to increase frequency of hand washing increased (not statistically significant). The majority of students sought information about the epidemic from Twitter (40\%), followed by television (36\%).

Conclusions: Nursing colleges may need to review existing or develop new epidemic-related policies. These include attendance of students in clinical settings and the duty of care towards patients during epidemics.

Key Words: Coronavirus, Nursing students, Policies, Education, Epidemics prevention, Nurses, Social media

\section{Introduction}

The Middle Eastern Coronavirus (MERS-CoV) is a serious and growing threat to communities around the world. A high proportion of people who have been affected by the virus are healthcare workers, patients who are admitted to hospital for other reasons, and visitors of patients. This strain of coronavirus that causes MERS-CoV was first reported in 2012 in Saudi Arabia. ${ }^{[1]}$ Since then, as of Nov 7, 2014, the World Health Organization has reported 909 laboratory confirmed infections, with 331 deaths. The epidemic has affected 22 countries in total. Most cases outside of the Middle East were identified as patients who had travelled to an endemic region and brought the virus back where they

\footnotetext{
* Correspondence: Bridget V. Stirling; Email: bstirling@pnu.edu.sa; Address: Princess Nourah University, Nursing Department Al-Iman Saud Bin Abdul Aziz Rd, Riyadh, KSA 84428, Saudi Arabia.
} 
were cared for in their own country. In Saudi Arabia, the epidemic is having a growing impact on healthcare workers as a number have been affected, and on the entire health care system. The WHO advice for all healthcare workers is to increase infection prevention and control measures to prevent the spread of MERS-CoV. ${ }^{[2]}$ As nurse educators, our faculty became concerned about the safety of our students in clinical practice. We were also concerned about the safety of our faculty, our student and faculty's families, and the other students that our students interact with when they are on campus.

Early identification of MERS CoV is difficult as initial symptoms are usually mild. A typical case might look something like the following: Fever and cough, followed by chills, sore throat, muscle pain, joint pain, and difficulty breathing. Severe progression includes pneumonia, Acute Respiratory Distress Syndrome (ARDS) and renal failure. ${ }^{[3]}$ In about a third of cases, there are also gastrointestinal symptoms such as vomiting and diarrhea. ${ }^{[4]}$ Identification of the virus can be made by nasopharyngeal swab. If the swab tests negative, a patient with a suspected case should be retested using lower respiratory specimens such as sputum, bronchoalveolar lavage, or endotracheal aspirate. While many treatments have been tried to reduce the effects of the virus, individual symptom management has been most successful. These include ventilator strategies for ARDS, treating co-infections, and renal replacement therapy for acute renal failure. Other strategies such as ribaviron, cyclosporine $\mathrm{A}$, and other treatments have been tried without clinical data to support their effectiveness. ${ }^{[5]}$

It has not yet been determined if there is a significant animal reservoir for MERS-CoV. However, antibodies have been found in camels, ${ }^{[6]}$ and the virus may have circulated for some time in bats. ${ }^{[7]}$ The microorganism that causes MERS$\mathrm{CoV}$ is an RNA virus. It spreads via the droplet route of transmission. Other factors that make MERS-CoV a potential global threat is that it has shown person-to-person transmission and transmission from patient to nurse, lacks a vaccine or any evidence of effectiveness of specific therapies, and results in a high mortality rate. ${ }^{[8]}$ The agent does not spread quickly, as the $\mathrm{R} 0$ or reproductive rate (the number of people that each infected person spreads it to on average, during the infectious period) is between 0.8 and $1.3 .^{[9]}$ Having an R0 of less than one usually means that the infection will die out in the long run. Having an R0 above one means that an outbreak can be sustained and therefore infection may be widespread. Therefore, in its present state, the infectivity of MERS-CoV is low. By comparison, the R0 for the 1918 pandemic influenza virus was between 2 and 3. ${ }^{[10]}$

The university where the study was conducted is a new allfemale university, located in Riyadh, the capital city of the Kingdom of Saudi Arabia. Riyadh is a growing city, currently home to almost 5.2 million people. ${ }^{[1]}$ Our nursing students fulfill their practice requirements at local hospitals

Published by Sciedu Press throughout the city. Many of the hospitals where our students were doing their clinical practice were serving confirmed cases of MERS-CoV.

\section{Method}

\subsection{Aim of the project and sampling}

The project had five specific aims. The aims were to identify the level of knowledge among nursing students, staff and faculty regarding the MERS-CoV epidemic, identify the level of concern about working with hospitals and patients with the virus among the different groups, identify gaps in their knowledge before and after the educational sessions, and identify student and staff current infection control behaviors (pre-test) and intention to change any behaviour as a result of the workshop (post-test).

\subsection{Design}

The design for this study included two surveys before and after educational workshops. The College of Nursing leadership appointed a small task force to come up with a response to the growing concern regarding MERS-CoV. The activities of the committee included reviewing the current literature, development of policies on attendance in clinical setting, and increasing infection control and health promotion messages throughout the college. Additional resources and supplies were requested. In preparation for the education workshop a Question \& Answer paper was prepared in the English language. The content of the presentation included patient profiling and current epidemiological evidence. Some of the student nurses were caring for patients at hospitals with confirmed coronavirus and all of the students and faculty were in close contact to potential cases. Therefore nursing students returning to the college community were at high risk for infection.

Research ethics approval was received from the College of Nursing, Ethics Committee. The questionnaires included a front page that asked for informed consent, in compliance with the University's policy. Two workshops were planned for nursing students and faculty. In addition to the informed consent, the participatory nature of the study was reinforced verbally, and some people chose not to participate.

The design of the workshop was based on the authors' lived experience, gained from involvement in other pandemic preparedness programs, as well as Best Practice from the literature. A PowerPoint presentation was prepared in the English language. The workshop material covered evidence from the literature about virus transmission, infectivity and preventative strategies. Presenters drew on their experience as clinical leads in previous pandemics. Students were directed to credible sources of further information. A detailed account of the patient-profiling to-date was presented. There was an opportunity for participants to ask questions. 
Participants were given 15 minutes at the beginning of the workshop to complete the initial questionnaire. The pretest consisted of four demographic questions to identify whether the participant was a student, staff member or faculty, whether they were currently caring for patients in the hospital, which hospital, and which semester they were in. The authors developed a questionnaire to understand the knowledge, attitudes and practice of students and faculty in light of the epidemic. The majority of questions were openended free-text questions (6/15 in the pre-test and 7/14 in the post-test). Others were nominal variables (yes/no, list of symptoms, or which semester etc.) and one question was a 5-point Likert scale ("extremely worried" to "not worried").

\subsection{Sampling and analytic strategy}

Requests to participate were sent out via e-mail to all students and faculty, with specific times for each group (undergraduates, faculty and intern students). Students were excused from regular classes and faculty who were unable to participate were asked to present similar evidence-based information to their students during their next class. Arrangements were made for interns who were working at a hospital with a perceived high number of cases of the virus, to attend a small, dedicated workshop to answer specific questions. Participation in the survey was completely voluntary.

All data was entered into Epi - Info ${ }^{[12]}$ (version 3.5.1). Summary statistics are presented as percentages of the participant group (student, intern or faculty) sample that responded to each question. Respondents with missing values were excluded from data summaries and statistical tests. $T$ tests were performed to assess the difference between means of the pre and post-test. This included the variables for frequency of hand washing and degree of worry or anxiety that the students were experiencing.

\section{Results}

Altogether, 65 students and 16 Faculty from the College of Nursing attended workshops about the MERS-CoV epidemic and not all chose to complete the pre and post-test. 25 student nurses chose to fill in pre-tests and 35 chose to participate in the post-tests. Student nurses who participated in the pre-test were all currently working in their clinical practice at local hospitals in Riyadh. 75\% of those who completed the post-test were in clinical practice. The majority of all students who participated were in their fourth year of education and therefore had more exposure to clinical settings. The students were representative of the population of students across the semesters in the program. Of 60 student responses, most were in semester 7 (30\%), followed by Interns (17\%) semesters 4 and 8 (both 15\%) and the rest were in semester 2 and 6 .

With regard to knowledge about MERS-CoV before the educational session, $40 \%$ knew that it had something to do with the respiratory system, $28 \%$ identified it as a virus and $28 \%$ stated that it was fatal. Other answers included that one of the symptoms was fever (20\%), that there was no vaccine $(12 \%)$ and that it had something to do with camels (12\%).

When asked about what they hoped to learn in the workshop, student nurses answered that they wanted to know about transmission (40\%), treatment and complications (32\%). Four students wanted to know everything about the epidemic. One student identified why it happened and another student wanted to know where it came from.

A higher number of student nurses in the pre-test identified feeling worry, anger, fear and confusion than the post-test. The percentage of students who had no feelings about the epidemic of MERS-CoV increased in the post-test (see Figure 1).

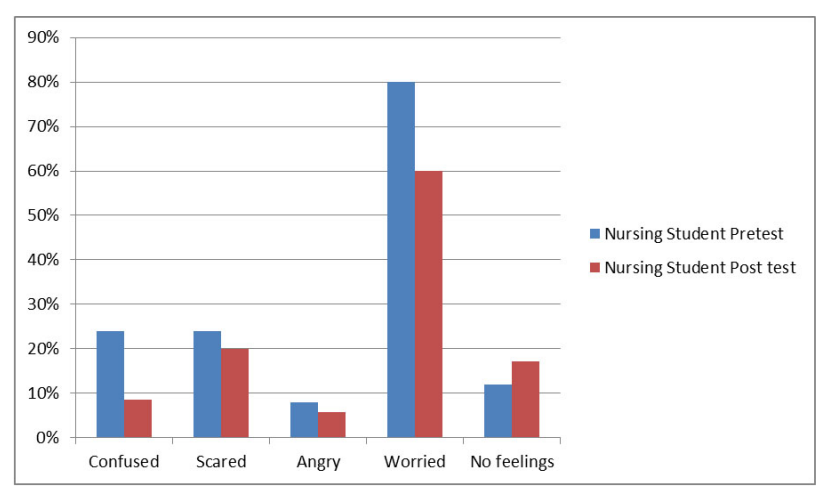

Figure 1: Nursing Students' Responses Regarding Feelings about the MERS-CoV Epidemic

With regards to their current level of anxiety, on a 5 point Likert scale, the majority of students during the pre-test reported a mean anxiety level of 3.13. An answer of 3 represented feeling "somewhat worried". During the post-test, the mean anxiety level was 2.61 for student nurses. The difference between the reduction of self-reported worry before the workshop and after was statistically significant ( $p$ is less than 0.05).

In the pre-test, advice for community members who experienced symptoms would include going to the hospital/doctor (32\%), using a facemask (20\%), hand hygiene (20\%) and others. In the post-test, student nurses stated that advice for community members who experienced symptoms would be going to the hospital/doctor (46\%), being tested (23\%), and isolating themselves at home (17\%).

Protective strategies for the students themselves that were identified in the pretest included the following: hand hygiene (32\%) and wear gown and N95 mask (16\%). Students in the pretest also mentioned not touching patients $(12 \%)$. In the post-test, nursing students identified hand hygiene (77\%), wearing an N95 mask (37\%) and keeping space between people and avoiding crowds (23\%). Hand 
washing frequency intention increased from what student nurses were currently doing (6.4 times per day) to 7.5 in the post-test.

When asked about MERS-CoV-like symptoms in the past 2 weeks, students responded that they had experienced fever (28\%), breathing problems $(28 \%)$, nausea and vomiting (12\%), achiness (4\%) and cough (20\%). Only 2 students had experienced none of the symptoms listed. Despite the high burden of symptoms in this nursing student population, and their proximity to patients during the epidemic only a single listed that they had sought testing (1 sputum and 1 blood test). Intended behaviour was measured in post-test questions regarding what students and faculty will do, should they experience MERS-CoV like symptoms. The most popular answer was go to the hospital for testing (35\%), followed by staying at home/isolating yourself $(17 \%)$.

Student nurses and faculty were asked about where they were getting their information about MERS-CoV. Student nurses were most likely to get their information from Twitter, followed by Internet news and friends/family. Faculty members looked to Internet news, followed by TV and friends and family. Social media is very important to young Saudi females, as these are methods of communications and feeling connected. Saudi Arabia is described as a digital society and leads the way in the use of Twitter in the Middle East with over 1.9 million Twitter users (Radcliffe, 2013) (see Figure 2\&3).

\section{Discussion}

Student nurses and Nursing faculty are among the key groups of people in any epidemic response. In the case of MERS-CoV, a serious pathogen with a case-fatality rate that has reached $60 \%$ (WHO, 2013) the virus is spread by droplet route or aerosolized through procedures to become airborne. Therefore, movement of people between areas where contact with the virus is highly probable (such as a hospital with known cases) and an area with a high density of vulnerable people (such as a university) should be limited. Whether students continue in clinical or not, their learning needs and resource needs must be addressed by the College faculty.

One of the main messages of the educational workshop was to "take MERS-CoV seriously, but don't panic". It was important for the students to be prepared to act both in the role of community educator and in their role as a learner. Students in close contact with patients are also moving in the university and classrooms, which are high-density environments.

In the post-test questionnaire a quarter of the students (26\%) learned about self-protection and how patients can be asymptomatic and $26 \%$ learned about transmission of the virus. All of faculty $(100 \%)$ learned about both routes of transmission and that there are asymptomatic carriers of the virus. All staff (100\%) stated they will change their behaviour after learning more about MERS CoV, $60 \%$ of students indicated changes of behaviour including increasing infection control by washing hands and uniforms and changing footwear inside and outside the hospital. When asked about intended behavior change, the most popular answer was that the student would change her behavior, but they did not give specific examples. Others identified hand washing more often, more care to how they washed their uniforms and carrying their hospital shoes back to their home in a separate plastic bag. Still others mentioned that they would choose to be less afraid and seek out new opportunities to learn about the epidemic from credible sources.

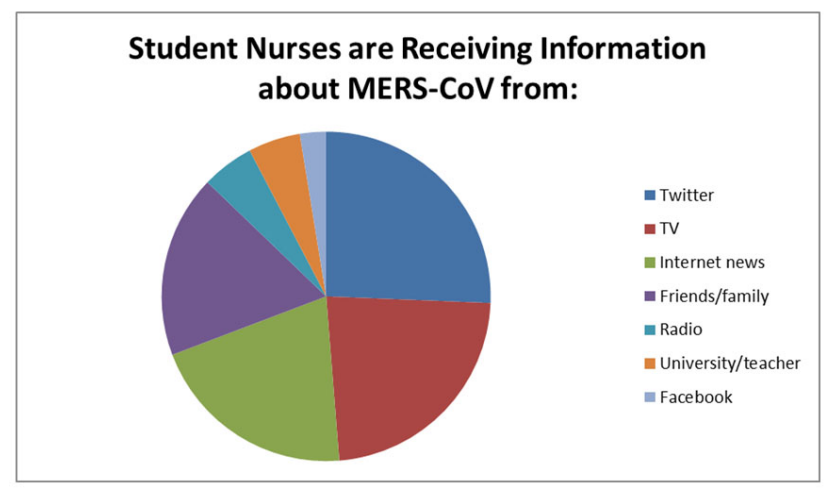

Figure 2: Where Student Nurses Receive Information about MERS-CoV

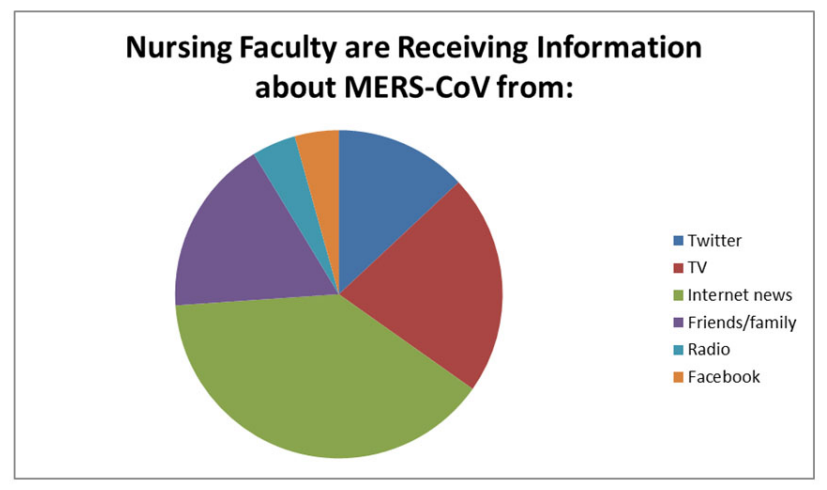

Figure 3: Where Nursing Faculty Receive Information about MERS-CoV

Hand washing frequency intention increased from what student nurses were currently doing (6.4 times per day) to 7.5 in the post-test. In observational studies ${ }^{[13]}$ health care workers washed their hands on average between 5 times and 30 times per shift. Even the intention to wash hands an average of 7.5 times per day, is still well below the average number of times a healthcare worker should be hand washing. This finding highlights an area for further reinforcement of the importance of hand hygiene. 
Nursing students also identified areas of learning that they needed more clarification about. There were still questions about possible vaccines. One student would have liked more information specifically about children in relation to MERSCoV. A follow-up e-mail has gone out to students reiterating the main messages from the workshop and addressing the concerns and questions that were identified as learning needs that had not been met.

Overall, the anxiety around the epidemic of MERS-CoV was reported to have decreased after the educational workshop. Worry by the students decreased significantly after the intervention. Students and faculty were better able to identify the mode of transmission and describe some tangible changes that they plan to make, given the information that was presented.

Students had been getting most of their information from social media, specifically Twitter. As a result, the researchers directed them to both the World Health Organization's Twitter access and also the Kingdom of Saudi Arabia's Twitter access. While social media can be an excellent way of communicating and being informed, we reiterated to the students and staff that they need to access credible sights with consistent data.

In conclusion, this study assisted nursing faculty in identifying areas of learning needs for students during an epidemic of a deadly respiratory infection. Student nurses may be an important conduit for the spread of infection and there is a great opportunity to prevent the spread of disease between the hospital and university populations. While student and faculty knowledge increased about disease transmission and prevention, the post-test results demonstrated that this effort should be the first step in a comprehensive review of the importance of appropriate hand washing frequency and use of Personal Protective Equipment. The difference between the self-reported worry before and after the workshop showed that the message of "take the epidemic seriously, but don't panic" may have been heard and followed by some students.

\section{Conflicts of Interest Disclosure}

The author declares that there is no conflict of interest statement.

\section{References}

[1] Zaki AM, van Boheemen S, Bestebroer TM, Osterhaus AD, Fouchier RA. Isolation of a novel coronavirus from a man with pneumonia in Saudi Arabia. N Engl J Med. 2012 Nov 8; 367(19): 181420. PMID:23075143 http://dx.doi .org/10.1056/NEJMoa121 1721

[2] WHO. Global Alert and Response. 2014. Available from: http: //www. who.int/csr/don/2014_04_16_mers/en/

[3] WHO. Mers CoV Research Group. 2013. Available from: http://www.who.int/csr/disease/coronavirus_infect ions/Update12_MERSCoV_update_22Nov13.pdf?ua=1

[4] Guery B, Poissy J, el Mansouf L et al. Clinical features and viral diagnosis of two cases of infection with Middle East Respiratory Syndrome coronavirus: a report of nosocomial transmission. Lancet. 2013; 381(9885): 2265-72. http://dx.doi.org/10.1016/S01 40-6736 (13) 60982-4

[5] Chan KH, Chan JF Tse H, Chen H, et al. Cross-reactive antibodies in convalescent SARS patients' sera against the emerging novel human coronavirus EMC (2012) by both immunofluorescent and neutralizing antibody tests. Journal of Infection. 2013; 67(2): 130140. PMID:23583636 http://dx.doi.org/10.1016/j.jinf . 2013.03.015

[6] Meyer, B, Müller, MA, Corman, VM, et al. Antibodies against MERS Coronavirus in Dromedaries, United Arab Emirates, 2003 and 2013. Emerging Infectious Diseases. 2013; 20(4): 552-559.
PMID:24655412 http://dx.doi.org/10.3201/eid2004.131 746

[7] Jie, C, Eden, J, Holmes, EC, Lin-Fa, W. Adaptive evolution of bat dipeptidyl peptidase 4 (dpp4): implications for the origin and emergence of Middle East respiratory syndrome coronavirus. Virology Journal. 2013; 10(1): 2-11.

[8] Arabi, YM, Arifi, AA, Balkhy, HH, et al. Clinical Course and Outcomes of Critically Ill Patients With Middle East Respiratory Syndrome Coronavirus Infection. Annals of Internal Medicine. 2002; 160(6): 389-401.

[9] Cauchemez S, Fraser C, Van Kerkhove MD, et al. Middle East respiratory syndrome coronavirus: quantification of the extent of the epidemic, surveillance biases, and transmissibility. Lancet Infect Dis. 2014 Jan; 14(1): 50-6. http://dx.doi.org/10.1016/S1473-3 099(13) 70304-9

[10] Mills, CE, Robins JM, and Lipsitch, M. Transmissibility of the 1918 pandemic influenza. 2004; Nature 432 7019: 904-6. PMID:15602562 http://dx.doi.org/10.1038/nature03063

[11] Saudi Arabia Government (2014) Ministry of Interior, Riyadh Principality 25 May 2014.

[12] Epi-info CDC- 3.5.1 Available from: http://wwwn.cdc.gov/epi info/

[13] Centre of Disease Control \& Prevention. Guidelines for Hand washing in healthcare settings: Recommendations of the Healthcare Infection Control Practices Advisory Committee and the HICPAC/SHEA/APIC/IDSA Hand Hygiene Task Force. 2002. Available from: http://www.cdc.gov/mmwr/preview/mmwrhtml/ rr5116a1.html 